Monatshefte für Chemie 107, 1265-1270 (1976)

by Springer-Verlag 1976

\title{
Derivate des \\ Sila-Mephenhydramins und Sila-Chlorphenoxamins
}

\author{
Sila-Pharmaka, 5. Mitt. ${ }^{1}$ \\ Von \\ Reinhold Tacke ${ }^{2}$ und Ulrich Wannagat \\ Institut für Anorganische Chemie, Technische Universität Braunschweig, \\ Bundesrepublik Deutschland \\ (Eingegangen am 10. Mai 1976) \\ Derivatives of Sila-Mephenhydramine and Sila-Chlorphenoxamine \\ Derivatives $\mathbf{A}$ and $\mathbf{B}$ of the two sila-antihistamines sila- \\ mephenhydramine and sila-chlorphenoxamine were synthesized \\ for the first time by the steps shown in scheme 1. They and their \\ precursors III and IV were characterized by their physical \\ (Table 1) and chemical properties and their structures confirmed \\ by NMR and mass spectroscopy (Tables 2 and 3). Their phar- \\ macological effects were investigated and compared with those \\ of the corresponding sila-antihistamines.
}

\section{Einführung}

In früheren Arbeiten ${ }^{3-5}$ haben wir über die Darstellung und Eigenschaften einiger Silicium-Analoga der Benzhydryläther-Antihistaminika des Struktur-Typs
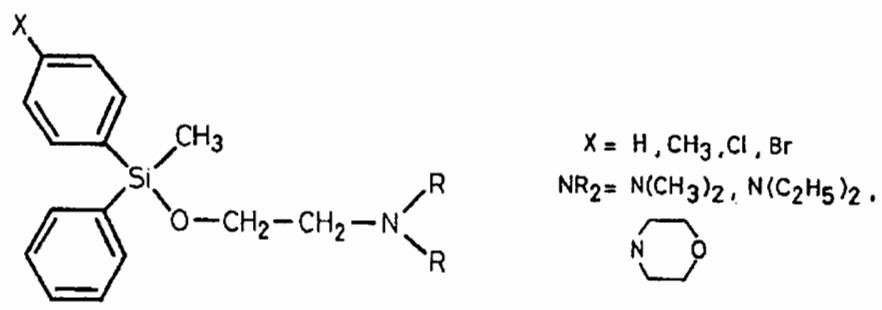

berichtet.

Die einzelnen Vertreter dieser über $X$ und $R$ variierten Verbindungsklasse zeigen ein weitgehend einheitliches pharmakologisches Wirkungsbild ${ }^{6}$. Die ED $_{50}$-Werte der untersuchten Wirkqualitäten werden durch die Substituenten $X$ und $\mathrm{R}$ nicht sehr stark beeinflußt. Die gefundenen Struktur-Wirkungsbeziehungen der Si-Verbindungen decken sich dabei weitgehend mit denen der C-Analoga. 
In Fortführung unserer Untersuchungen an Sila-Benzhydryläthern wurden zusätzliche Änderungen am oben genannten Struktur-Typ vorgenommen und untersucht:

1. Verlängerung der Aminoalkoxygruppe im Sila-Mephenhydramin $\left(X=\mathrm{H}, \mathrm{R}=\mathrm{CH}_{3}\right)$ um eine $\mathrm{CH}_{2}$-Gruppe $(\rightarrow \mathrm{A})$.

Skizze 1. Zur Darstellung der Derivate A und B des Sila-Mephenhydramins und Sila-Chlorphenoxamins

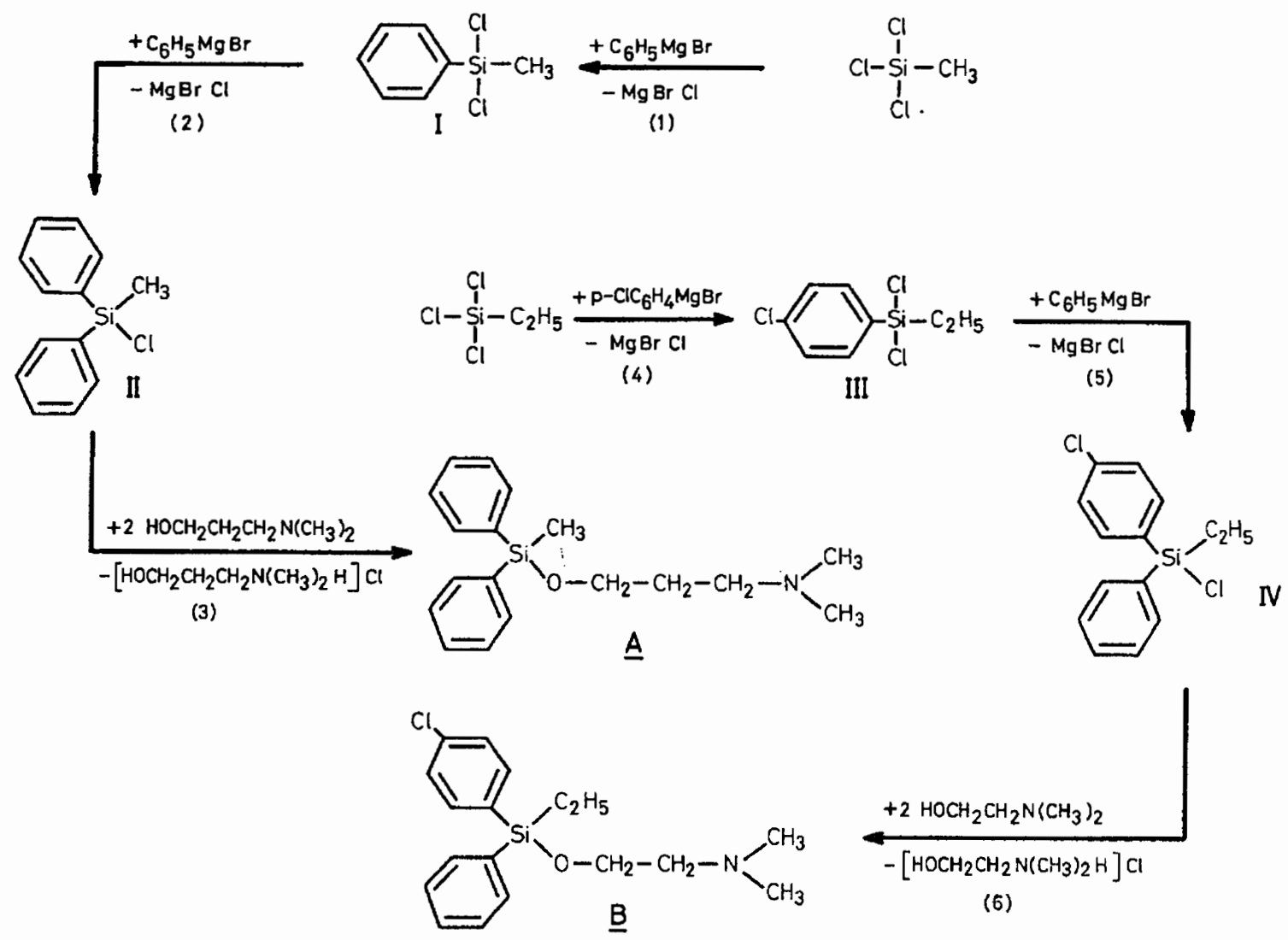

2. Austausch der am Si-Atom gebundenen Methylgruppe im SilaChlorphenoxamin $\left(X=\mathrm{Cl}, \mathrm{R}=\mathrm{CH}_{3}\right)$ durch eine Äthylgruppe $(\rightarrow \mathbf{B})$.

Über die Darstellung und Eigenschaften entsprechender Verbin. dungen wird im folgenden berichtet.

2. Zur Darstellung der neuen Sila-Benzhydryläther und ihrer Vorstufen

A wurde, von Methyltrichlorsilan ausgehend, nach bekanntem Verfahren durch Umsetzung mit Phenylmagnesiumbromid (Rkk. 1 und 2) in zwei Stufen und anschließende Umsetzung mit 3-Dimethylaminopropanol (Rk. 3) erhalten (siehe Skizze 1). 
Analog wurde B dargestellt: Die Umsetzung von Äthyltrichlorsilan mit p-Chlorphenylmagnesiumbromid (Rk. 4) führt zunächst zu III, das sich durch erneute Behandlung mit Phenylmagnesiumbromid (Rk. 5) in IV überführen läßt. Dieses reagiert mit 2-Dimethylaminoäthanol (Rk. 6) zu B (siehe Skizze).

\section{Experimenteller Teil}

Die Darstellung von I-IV sowie A und $\mathbf{B}$ erfolgte in der gleichen Weise wie bei den in früheren Arbeiten ${ }^{3-5}$ beschriebenen analogen Verbindungen. Für exper. Details sei auf ${ }^{2-4}$ verwiesen.

Tabelle 1. Physikalische und elementaranalytische ${ }^{\mathrm{b}}$ Daten der neu dargestellten Verbindungen

\begin{tabular}{|c|c|c|c|c|c|c|c|c|}
\hline $\begin{array}{l}\text { Lfd. } \\
\text { Nr. }\end{array}$ & $\begin{array}{l}\text { Summen- } \\
\text { formel }\end{array}$ & $\begin{array}{l}\text { Molgew. } \\
\text { ber. }^{2}\end{array}$ & {$\left[{ }^{\circ} \mathrm{C}\right]^{\mathrm{Sd}}$} & $\begin{array}{l}\text { p. } \\
\text { [Torr] }\end{array}$ & $D_{4}^{20}$ & $n_{\mathrm{D}}^{20}$ & $\begin{array}{l}\text { \% C } \\
\text { ber. gef. }\end{array}$ & $\begin{array}{c}\% \mathrm{H} \\
\text { ber. gef. }\end{array}$ \\
\hline III & $\mathrm{C}_{8} \mathrm{H}_{9} \mathrm{Cl}_{3} \mathrm{Si}$ & 239,61 & 109 & 5 & 1,2551 & 1,5346 & $40,1039,50$ & $3,79 \quad 3,45$ \\
\hline IV & $\mathrm{C}_{14} \mathrm{H}_{14} \mathrm{Cl}_{2} \mathrm{Si}$ & 281,26 & 115 & 0,1 & 1,1724 & 1,5783 & $59,7959,80$ & $5,02 \quad 5,01$ \\
\hline A & $\mathrm{C}_{18} \mathrm{H}_{25} \mathrm{NOSi}$ & 299,49 & $134-135$ & 0,4 & 0,9987 & 1,5352 & $72,19 \quad 71,42$ & $8,41 \quad 8,22$ \\
\hline $\mathbf{B}$ & $\mathrm{C}_{18} \mathrm{H}_{24} \mathrm{ClNOSi}$ & 333,94 & $128-129$ & 0,05 & 1,0738 & 1,5468 & $64,74,65,20$ & $7,24 \quad 7,45$ \\
\hline
\end{tabular}

p-Chlorphenyl-äthyl-dichlorsilan (III): Gemäß Rk. (4) durch Zutropfen einer Lösung von $0,4 \mathrm{Mol} p$-Chlorphenylmagnesiumbromid in $360 \mathrm{ml}$ Diäthyläther $(=\ddot{A})$ zu $65,4 \mathrm{~g}(0,4 \mathrm{Mol})$ Äthyltrichlorsilan in $200 \mathrm{ml} \ddot{A}$. Ausb. 46 g III ( $48 \%)$.

p-Chlorphenyl-äthyl-phenyl-chlorsilan (IV): Gemäß Rk. (5) durch Zutropfen einer Lösung von $0,2 \mathrm{Mol}$ Phenylmagnesiumbromid in $180 \mathrm{ml} \ddot{A}$ zu 47,9 g (0,2 Mol) III in $100 \mathrm{ml} \ddot{A}$. Ausb. 25,9 g IV (46\%).

$N, N$-Dimethyl-3-(methyl-diphenylsilyloxy)-propylamin (A): GemäßRk. (3) durch Zutropfen einer Lösung von 23,3 g (0,1 Mol) II in $150 \mathrm{ml}$ Petroläther $\left(40-65^{\circ} \mathrm{C}=P \ddot{A}\right) \mathrm{zu} 61,9 \mathrm{~g}(0,6 \mathrm{Mol}) 3$-Dimethylaminopropanol in $200 \mathrm{ml}$ $P \ddot{A}$. Ausb. $23 \mathrm{~g} \mathrm{~A}(76,8 \%)$.

$N, N$-Dimethyl-2-(p-chlorphenyl-äthyl-phenylsilyloxy)-äthylamin (B): Gemäß Rk. (6) durch Zutropfen einer Lösung von $28,1 \mathrm{~g}(0,1 \mathrm{Mol}) \mathrm{IV}$ in $150 \mathrm{ml}$ $P \ddot{A}$ zu $53,5 \mathrm{~g}(0,6 \mathrm{Mol}) 2$-Dimethylaminoäthanol in $200 \mathrm{ml} P \ddot{A}$. Ausb. $26,4 \mathrm{~g}$ B $(79 \%)$.

3. Chemische und physikalische Eigenschaften sowie Strukturermittlung

Die Vorstufen III und IV (Eigenschaften von I und II siehe in ${ }^{3}$ ) sind wasserklare, hydrolyseempfindliche Flüssigkeiten, die sich gut in den gän. gigen inerten organischen Solventien lösen. 
III und IV wurden bisher noch nicht in der Literatur beschrieben. Es wird lediglich ein Gemisch von isomeren Verbindungen des Typs $\mathrm{C}_{2} \mathrm{H}_{5}\left(\mathrm{ClC}_{6} \mathrm{H}_{4}\right) \mathrm{SiCl}_{2}$ erwähnt ${ }^{7}$, das aus einer Chlorierung von $\mathrm{C}_{2} \mathrm{H}_{5}\left(\mathrm{C}_{6} \mathrm{H}_{5}\right) \mathrm{SiCl}_{2}$ hervorgegangen ist.

$\mathbf{A}$ und $\mathbf{B}$ sind ölige Flüssigkeiten, die sich ebenfalls gut in den üblichen inerten organischen Lösungsmitteln lösen. Die Wasserlöslichkeit und das Hydrolyseverhalten (Spaltung der Si-OC-Bindung) dieser Verbindungen ist ähnlich wie bei den schon früher von uns beschriebenen Sila-benzhydryläthern ${ }^{3-5}$.

Tabelle 2. ${ }^{1} \mathrm{H}-\mathrm{NMR}-S p e l t r e n$ der neu dargestellten Verbindungen. Chemische Verschiebungen in $\delta[p p m]$, Lösungsmittel: $\mathrm{CCl}_{4}$, Standard intern: TMS

\begin{tabular}{llccl}
\hline Lfd. Nr. & \multicolumn{1}{c}{$\delta$} & Multiplizität & rel. Intens. & \multicolumn{1}{c}{ Zuordnung } \\
\hline \multirow{2}{*}{ III } & $0,7-1,5$ & $\mathrm{~m}$ & 5 & $\mathrm{SiC}_{2} \mathrm{H}_{5}$ \\
& $7,47 \mathrm{a}$ & $\mathrm{m}$ & 4 & $\mathrm{SiC}_{6} \mathrm{H}_{4} \mathrm{Cl}$ \\
IV & $0,7-1,5$ & $\mathrm{~m}$ & 5 & $\mathrm{SiC}_{2} \mathrm{H}_{5}$ \\
& $7,0-7,8$ & $\mathrm{~m}$ & 9 & $\mathrm{SiC}_{6} \mathrm{H}_{5}, \mathrm{SiC}_{6} \mathrm{H}_{4} \mathrm{Cl}$ \\
A & 0,58 & $\mathrm{~s}$ & 3 & $\mathrm{SiCH}_{3}$ \\
& $1,3-2,5$ & $\mathrm{~m}$ & 10 & $\mathrm{CCH}_{2} \mathrm{CH}_{2} \mathrm{~N}$ \\
& 2,06 & $\mathrm{~s}$ & 3 & $\mathrm{~N}_{\left(\mathrm{CH}_{3}\right)_{2}}$ \\
& 3,69 & $\mathrm{t}$ & 2 & $\mathrm{OCH}_{2} \mathrm{C}$ \\
& $7,0-7,8$ & $\mathrm{~m}$ & 10 & $\mathrm{Si}_{6}\left(\mathrm{C}_{6} \mathrm{H}_{5}\right)_{2}$ \\
B & $1,0-1,5$ & $\mathrm{~m}$ & 5 & $\mathrm{SiC}_{2} \mathrm{H}_{5}$ \\
& 2,10 & $\mathrm{~s}$ & 6 & $\mathrm{~N}_{\left(\mathrm{CH}_{3}\right)_{2}}$ \\
& 2,38 & $\mathrm{t}$ & 2 & $\mathrm{CCH}_{2} \mathrm{~N}$ \\
& 3,69 & $\mathrm{t}$ & 2 & $\mathrm{OCH}_{2} \mathrm{C}$ \\
& $7,0-7,7$ & $\mathrm{~m}$ & 9 & $\mathrm{SiC}_{6} \mathrm{H}_{5}, \mathrm{SiC}_{6} \mathrm{H}_{4} \mathrm{Cl}$
\end{tabular}

a Zentrum des $A A^{\prime} B B^{\prime}$-Systems.

Zur Strukturermittlung von III, IV, A und B wurden neben den Elementaranalysen vor allem ${ }^{1} \mathrm{H}$-NMR-Spektren und Massenspektren herangezogen. Die jeweiligen spektroskopischen Daten, die den in früheren Mitteilungen beschriebenen Spektren weitgehend entsprechen, sind in Tab. 2 und Tab. 3 aufgeführt.

4. Pharmakologische Eigenschaften der Sila-benzhydryläther A und B

Die pharmakologischen Untersuchungen wurden in enger Zusammenarbeit am Institut für Pharmakologie und Toxikologie der Technischen Universität Braunschweig von H.-U. Rossée und F. Meyer durchgeführt. Für ausführliche Informationen sei auf die Dissertation Rossée verwiesen ${ }^{6}$. 
In-vitro-Versuche am isolierten Meerschweinchen-Ileum ergaben, da $B$ die neu dargestellten Verbindungen $\mathbf{A}$ und $\mathbf{B}$ sowohl histaminolytische als auch anticholinerge und muskulotrop spasmolytische Eigenschaften besitzen ${ }^{6}$. Der Vergleich der entsprechenden $\mathrm{ED}_{50}$-Werte von

Tabelle 3. Einige charakteristische Schlüsselbruchstücke aus den Massenspektren der neu dargestellten Verbindungen. Probeneinführung durch Hochtemperatureinlaß (III) und Direkteinla $\beta$ (IV, A, B), Elektronenstoßionisierung mit $70 \mathrm{eV}$

\begin{tabular}{|c|c|c|c|c|}
\hline $\begin{array}{l}\text { Lfd. } \\
\text { Nr. }\end{array}$ & $m / e$ & $\begin{array}{l}\text { relative I } \\
\text { Intensität }\end{array}$ & $\begin{array}{l}\text { Isotopen- } \\
\text { muster }\end{array}$ & Ion \\
\hline III & $\begin{array}{l}238 / 240 / 242 / 244 \\
209 / 211 / 213 / 215 \\
63 / 65\end{array}$ & $\begin{array}{l}17 / 17 / 6 / 1 \\
99 / 100 / 35 / 6 \\
30 / 11\end{array}$ & $\begin{array}{l}\mathrm{Cl}_{3} \\
\mathrm{Cl}_{3} \\
\mathrm{Cl}\end{array}$ & $\begin{array}{l}M^{+} \\
M^{+}-\mathrm{C}_{2} \mathrm{H}_{5}{ }^{\mathrm{a}} \\
\mathrm{SiCl}^{+}\end{array}$ \\
\hline IV & $\begin{array}{l}280 / 282 / 284 \\
251 / 253 / 255 \\
63 / 65\end{array}$ & $\begin{array}{l}15 / 13 / 3 \\
100 / 67 / 15 \\
34 / 12\end{array}$ & $\begin{array}{l}\mathrm{Cl}_{2} \\
\mathrm{Cl}_{2} \\
\mathrm{Cl}\end{array}$ & $\begin{array}{l}M^{+} \\
M^{+}-\mathrm{C}_{2} \mathrm{H}_{5} \mathrm{~b} \\
\mathrm{SiCl}^{+}\end{array}$ \\
\hline $\mathbf{A}$ & $\begin{array}{l}299 \\
284 \\
199 \\
197 \\
105 \\
77 \\
58\end{array}$ & $\begin{array}{l}24 \\
1 \\
3 \\
10 \\
5 \\
3 \\
100\end{array}$ & & $\begin{array}{l}M^{+} \\
M^{+}-\mathrm{CH}_{3} \\
\mathrm{Si}\left(\mathrm{C}_{6} \mathrm{H}_{5}\right)_{2} \mathrm{OH}^{+} \\
M^{+}-\mathrm{OCH}_{2} \mathrm{CH}_{2} \mathrm{CH}_{2} \mathrm{~N}\left(\mathrm{CH}_{3}\right)_{2} \\
\mathrm{SiC}_{6} \mathrm{H}_{5}{ }^{+} \\
\mathrm{C}_{6} \mathrm{H}_{5}+ \\
\mathrm{CH}_{2}=\stackrel{+}{\mathrm{N}}\left(\mathrm{CH}_{3}\right)_{2}\end{array}$ \\
\hline $\mathbf{B}$ & $\begin{array}{l}333 / 335 \\
304 / 306 \\
245 / 247 \\
233 / 235 \\
105 \\
77 \\
58\end{array}$ & $\begin{array}{l}17 / 5 \\
7 / 2 \\
3 / 1 \\
16 / 5 \\
16 \\
7 \\
100\end{array}$ & $\begin{array}{l}\mathrm{Cl} \\
\mathrm{Cl} \\
\mathrm{Cl} \\
\mathrm{Cl}\end{array}$ & $\begin{array}{l}M^{+} \\
M^{+}-\mathrm{C}_{2} \mathrm{H}_{5} \\
M^{+}-\mathrm{OCH}_{2} \mathrm{CH}_{2} \mathrm{~N}\left(\mathrm{CH}_{3}\right)_{2} \\
\mathrm{Si}\left(\mathrm{C}_{6} \mathrm{H}_{4} \mathrm{Cl}\right)\left(\mathrm{C}_{6} \mathrm{H}_{5}\right) \mathrm{OH}^{+} \\
\mathrm{SiC}_{6} \mathrm{H}_{5}+ \\
\mathrm{C}_{6} \mathrm{H}_{5}^{+} \\
\mathrm{CH}_{2}=\stackrel{+}{\mathrm{N}}\left(\mathrm{CH}_{3}\right)_{2}\end{array}$ \\
\hline
\end{tabular}

a Der Übergang von $M^{+} \mathrm{zu}\left(M^{+}-\mathrm{C}_{2} \mathrm{H}_{5}\right)$ ist durch entsprechende Übergangssignale bei $m / e=183,5 / 185,5 / 187,5 / 189,4$ belegt.

b Der Übergang von $M^{+} \mathrm{zu}\left(M^{+}-\mathrm{C}_{2} \mathrm{H}_{5}\right)$ ist dureh entsprechende Übergangssignale bei $m / e=225 / 227 / 229$ belegt.

Sila-Mephenhydramin und A bzw. von Sila-Chlorphenoxamin und B ergibt, daß die durchgeführten Strukturabwandlungen auf die anticholinerge und muskulotrop spasmolytische Wirkung praktisch keinen Einfluß haben. Dagegen ist die histaminolytische Aktivität von A und B im Vergleich zum Sila-Mephenhydramin bzw. Sila-Chlorphenoxamin erheblich abgeschwächt. Der Histamin-Antagonismus nimmt sowohl durch die Verlängerung der Aminoalkoxygruppe als auch durch die 
Äthylsubstitution am Si-Atom stark ab (1:20 bzw. 1: 75). Der letztgenannte Befund steht im Einklang mit einer analogen Struktur-Wirkungsbeziehung ${ }^{8}$ zwischen dem Chlorphenoxamin und dessen entsprechendem Äthylderivat.

Auf Grund ihres hydrolytischen Abbaus ist die Bioaktivität von A und B nur von kurzer Dauer (etwa 16 Min., gemessen in Tyrodelösung

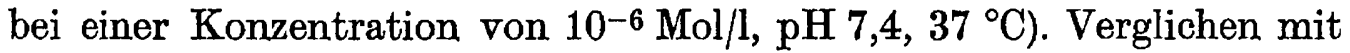
dem Sila-Mephenhydramin hat $\mathbf{A}$ eine um etwa $21 \%$ verkürzte Wirkung, B wirkt dagegen etwa 5\% länger als das Sila-Chlorphenoxamin.

Unser Dank gilt dem Verband der Chemischen Industrie, Frankfurt am Main, für die Unterstützung mit Sachmitteln, der Bayer-AG, Leverkusen, für die Überlassung von Chlorsilanen, sowie Herrn Dr. H.-U. Rossée und Herrn Prof. Dr. F. Meyer für die pharmakologischen Untersuchungen an den neu dargestellten Verbindungen.

\section{Literatur}

1 4. Mitt.: $R$. Tacke und U. Wannagat, 107, 449 (1976).

2 Mit Auszügen aus der Dissertation R. Tacke, Techn. Univ. Braunschweig 1974.

3 R. Tacke und U. Wannagat, Mh. Chem. 106, 1005 (1975).

4 R. Tacke und $U$. Wannagat, Mh. Chem. 107, 111 (1976).

5 R. Tacke und $U$. Wannagat, Mh. Chem. 107, 439 (1976).

6 Dissertation H.-U. Rossée, Techn. Univ. Braunschweig 1974.

7 G. V. Motsarev, V.T. Inshakova, V.I.Kolbasov und R.V.Dzhagatspanyan, Zh. Obshch. Khim. 41, 1573 (1971); Chem. Abstr. 75, 129871 (1971).

8 H. Arnold, N. Brock, E. Kühas und D. Lorenz, Arzneimittel-Forsch. 4, 189 (1954).

Korrespondenz und Sonderdrucke:

Dr. R. Tacke

Institut für Anorganische Chemie

Technische Universität Braunschweig

Pockelsstraße 4

D-3300 Braunschweig

Bundesrepublik Deutschland 
Mona.tshefte für Chemie 107, 1271-1279 (1976)

O by Springer-Verlag 1976

\title{
Isoelektronische Derivate des Sila-Clofenetamins und des Sila-Mebrophenhydramins
}

\author{
(Sila-Pharmaka, 6. Mitt. ${ }^{1}$ ) \\ Von \\ Reinhold Tacke ${ }^{2}$ und Ulrich Wannagat \\ Institut für Anorganische Chemie, Technische Universität Braunschweig, \\ Bundesrepublik Deutschland
}

(Eingegangen am 10. Mai 1976)

\begin{abstract}
Isoelectronic Derivatives of Sila-Clofenetamine and Sila-Mebrophenhydramine
\end{abstract}

\begin{abstract}
Isoelectronic derivatives (A and $B$ ) and a homolog (C) of the two sila-antihistamines sila-clofenetamine and silamebrophenhydramine were synthesized for the first time by the steps shown in scheme 1. They and their unknown precursors II-IV were characterized by their physical (Table 1) and chemical properties and their structures confirmed by ${ }^{1} \mathrm{H}-\mathrm{NMR}$ and mass spectroscopy (Tables 2 and 3 ). The pharmacological effects of $\mathbf{A}$ and $\mathbf{B}$ were investigated and compared with those of the corresponding $\mathrm{O}$-isosteric sila-antihistamines (Chapter 5).
\end{abstract}

\section{Einführung}

Im Rahmen unserer Arbeiten über Sila-Pharmaka konnten wir bereits früher verschiedene Silicium-Analoga der BenzhydrylätherAntihistaminika ${ }^{3-5}$ synthetisieren. Die bisher untersuchten Vertreter dieser Verbindungs-Klasse $\left[X=\mathrm{CH}_{3}, \mathrm{H}, \mathrm{Cl}, \mathrm{Br}\right.$ und $\mathrm{NR}_{2}=\mathrm{N}\left(\mathrm{CH}_{3}\right)_{2}$, $\mathrm{N}\left(\mathrm{C}_{2} \mathrm{H}_{5}\right)_{2}$, Morpholino] besitzen $u$. a. histaminolytische, anticholinerge

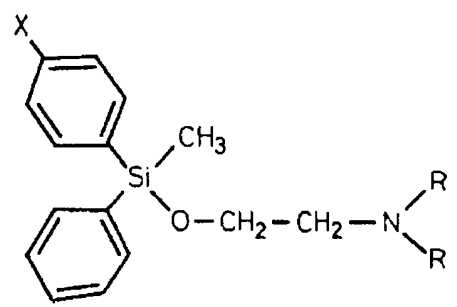

und muskulotrop spasmolytische Eigenschaften ${ }^{6}$, wobei die erstgenannte Wirkqualität stets am stärksten ausgeprägt ist. 
Während die pharmakologischen Wirkungen durch Variation von $X$ und $\mathrm{R}$ nur wenig beeinflußt werden, führen andere Strukturabwandlungen $\mathrm{zu}$ starken Veränderungen. So bedingt sowohl eine NQuaternierung ${ }^{7}$ als auch ein Austausch einer Phenylgruppe gegen eine Norbornenylgruppe ${ }^{8}$ einen deutlichen Verlust an histaminolytischer Aktivität und einen starken Gewinn anticholinerger Wirkung. Die Verlängerung der Aminoalkoxygruppe ${ }^{1}$ durch eine $\mathrm{CH}_{2}$-Gruppe sowie die Substitution ${ }^{1}$ einer $\mathrm{Si}-\mathrm{CH}_{3}$ - durch eine $\mathrm{Si}-\mathrm{C}_{2} \mathrm{H}_{5}$-Gruppe führt ebenfalls zu einer erheblichen Abschwächung der histaminolytischen Aktivität, während die beiden anderen Wirkqualitäten hierdurch nur wenig verändert werden.

Allen bisher dargestellten Sila-benzhydryläthern und ihren Derivaten ist eine kurze Wirkungsdauer $(<30$ Min.) gemeinsam, da die Verbindungen an der $\mathrm{Si}-\mathrm{O}-\mathrm{C}-$ Bindung hydrolytisch gespalten werden.

Skizze 1. Zur Darstellung der isoelektronischen Derivate A und B des Sila-Clofenetamins und des Sila-Mebrophenhydramins

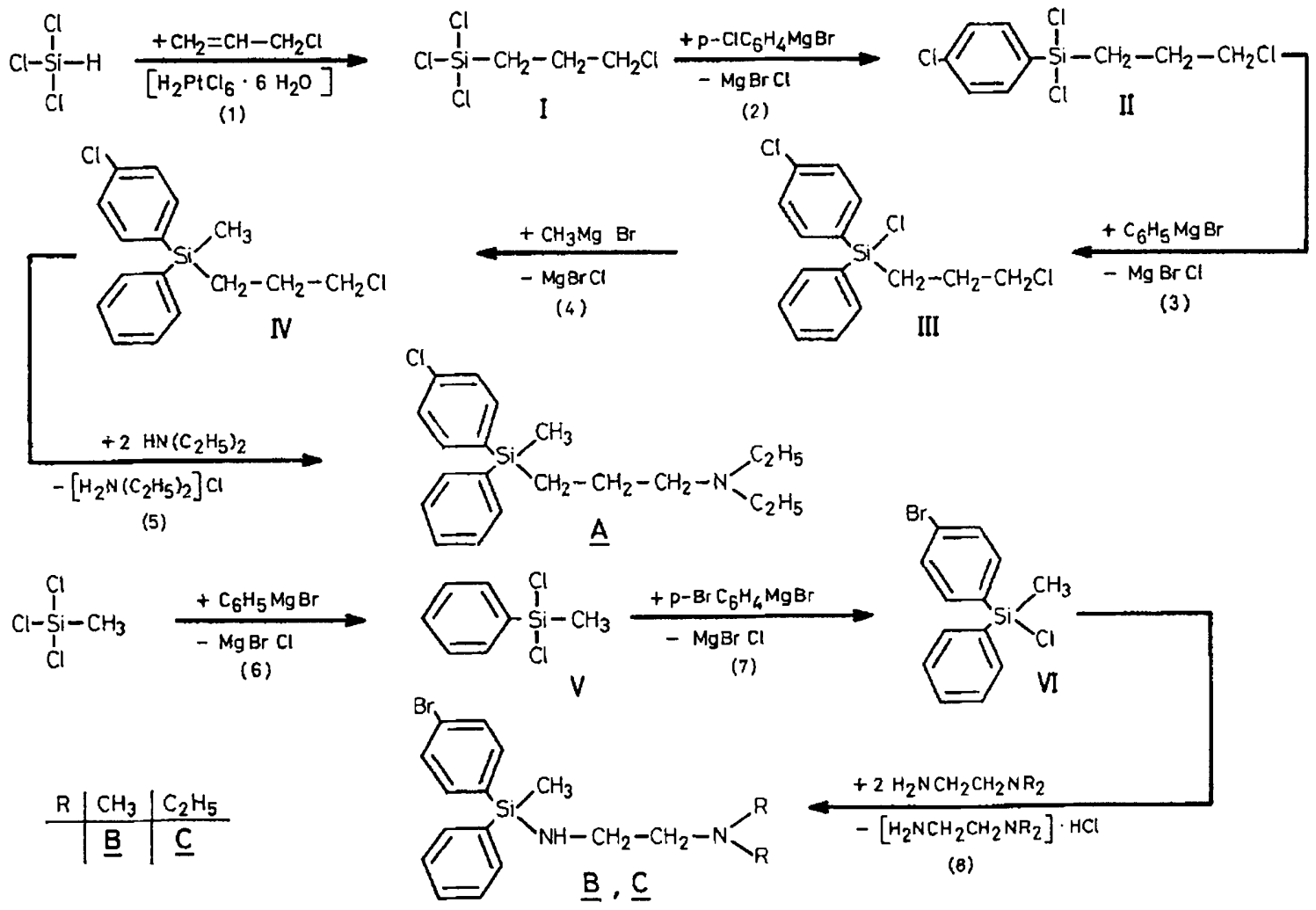

In Fortführung unserer Untersuchungen an Sila-benzhydryläthern haben wir weitere Veränderungen am Strukturgerüst dieser Verbindungen vorgenommen und in Hinblick auf die biologische Aktivität und das Hydrolyseverhalten untersucht. Im folgenden soll über die 
Darstellung und Eigenschaften von isoelektronischen Derivaten des Sila-Clofenetamins $\left(X=\mathrm{Cl}, \mathrm{R}=\mathrm{C}_{2} \mathrm{H}_{5}\right)$ und Sila-Mebrophenhydramins $\left(X=\mathrm{Br}, \mathrm{R}=\mathrm{CH}_{3}\right)$ berichtet werden, bei denen das O-Atom durch eine $\mathrm{CH}_{2}$-Gruppe $(\rightarrow \mathrm{A})$ bzw. eine NH-Gruppe $(\rightarrow \mathbf{B})$ ersetzt ist.

2. Die Darstellung der isosteren Sila-benzhydrylätherDerivate A, B und $\mathbf{C}$ sowie ihrer Vorstufen

Zur Darstellung von A wurde zunächst nach bekanntem Verfahren ${ }^{9}$ durch Addition (Rk. 1) von Allylchlorid an Trichlorsilan (in Gegenwart katalytischer Mengen $\mathrm{H}_{2} \mathrm{PtCl}_{6} \cdot 6 \mathrm{H}_{2} \mathrm{O}$ ) 3-Chlorpropyl-trichlorsilan (I) synthetisiert. Dieses ließ sich durch Umsetzung mit p-Chlorphenylmagnesiumbromid (Rk. 2) in II und durch weitere Reaktion mit Phenylmagnesiumbromid (Rk. 3) in III überführen. Durch eine letzte Grignardstufe mit Methylmagnesiumbromid (Rk.4) entstand IV, das durch Umsetzung mit Diäthylamin im Bombenrohr (Rk.5) schließlich A ergab (s. Skizze 1).

Zur Darstellung von. B und $\mathbf{C}$ wurde, von Methyltrichlorsilan ausgehend, nach bekanntem Verfahren durch stufenweise Reaktion mit Phenylmagnesiumbromid (Rk.6) ${ }^{10}$ und p-Bromphenylmagnesiumbromid (Rk. 7) ${ }^{11}$ über $\mathrm{V}$ zunächst VI synthetisiert. Dieses reagierte gemäß Rk. (8) mit den entsprechenden N,N-Dialkyläthylendiaminen zu B bzw. C (s. Skizze 1).

\section{Experimenteller Teil}

Die Darstellung von I, V und VI erfolgte in Anlehnung an die angegebenen Literaturzitate.

Die Synthese von II-IV gelang in der üblichen Weise durch Grignardreaktionen, die problemlos abliefen; bei der Aufarbeitung traten aber Schwierigkeiten auf, da sich die Verbindungen bei der Destillation aus den Rohprodukten z. T. durch $\gamma$-Eliminierung zersetzten (besonders III und IV). So wurde z. B. bei der Vakuumdestillation von III aus dem Rohprodukt mehrfach eine fast quantit. Zersetzung zu p-Chlorphenyl-phenyldichlorsilan beobachtet. Durch eine möglichst vollständige Entfernung der Mg-Salze und von nicht umgesetztem Grignardreagens (durch Fällung mit $T H F$ und $P \ddot{A}$ und nachfolgende Filtration über wasserfr. $\mathrm{Na}_{2} \mathrm{SO}_{4}$ ) konnte die $\gamma$-Eliminierung jedoch weitgehend unterdrückt werden. Zur Isolierung von reinem IV erwies es sich außerdem als nützlich, vor der Destillation etwas Diäthylamin zuzugeben.

\section{3-Chlorpropyl-(p-chlorphenyl)-dichlorsilan (II)}

Gemäß Rk. (2) tropft man zu $212 \mathrm{~g}$ (1 Mol) I in $500 \mathrm{ml}$ Diäthyläther ( $=\ddot{A}$ ) eine Lösung von $1 \mathrm{Mol} \mathrm{p}$-Chlorphenylmagnesiumbromid in $800 \mathrm{ml}$ $\ddot{A}$, rührt 2 Stdn. unter Rückfluß weiter, tropft anschließend in der Hitze langsam $200 \mathrm{ml} T H F$ hinzu, rührt $12 \mathrm{Stdn}$. bei $20^{\circ} \mathrm{C}$, filtriert von den $\mathrm{Mg-Salzen,} \mathrm{zieht} \mathrm{das} \mathrm{Lösungsmittel} \mathrm{bei} 20^{\circ} \mathrm{C}$ im Vak. möglichst vollständig 
ab, nimmt den Rückstand in $600 \mathrm{ml}$ Petroläther $\left(40 / 65^{\circ} \mathrm{C}=P \ddot{A}\right)$ auf, filtriert über eine Schicht von wasserfr. $\mathrm{Na}_{2} \mathrm{SO}_{4}$ und zieht das Lösungsmittel bei $20^{\circ} \mathrm{C}$ im Vak. vollständig ab. Anschließend nimmt man den Rückstand erneut in $600 \mathrm{ml} P \ddot{A}$ auf und filtriert nochmals über $\mathrm{Na}_{2} \mathrm{SO}_{4}$, zieht das Lösungsmittel ab und destilliert im Vak. über eine Vigreux-, anschließend über eine Drehbandkolonne. Ausb. 132,5 g II (46\%).

\section{3-Chlorpropyl-(p-chlorphenyl)-phenyl-chlorsilan (III)}

Gemäß Rk. (3) durch Zutropfen einer Lösung von $0,2 \mathrm{Mol} \mathrm{C}_{6} \mathrm{H}_{5} \mathrm{MgBr}$ in $160 \mathrm{ml} \ddot{A} \mathrm{zu} 57,6 \mathrm{~g}(0,2 \mathrm{Mol})$ II in $100 \mathrm{ml} \ddot{A}$. Reaktionsbedingungen und Aufarbeitung analog zu Rk. (2). Ausb. 26,4 g III (40\%).

\section{3-Chlorpropyl-(p-chlorphenyl)-methyl-phenylsilan (IV)}

Gemäß Rk. (4) durch Zutropfen einer Lösung von $0,1 \mathrm{Mol} \mathrm{CH}_{3} \mathrm{MgBr}$ in $200 \mathrm{ml} \ddot{A}$ zu $33 \mathrm{~g}(0,1 \mathrm{Mol})$ III in $50 \mathrm{ml} \ddot{A}$. Anschließend 2 Stdn. Rückfluß, Fällung von Mg-Salzen mit $50 \mathrm{ml} T H F$, $12 \mathrm{Stdn}$. Rühren bei $20^{\circ} \mathrm{C}$, Filtration von den $\mathrm{Mg}$-Salzen, Abziehen des Lösungsmittels bei $20^{\circ} \mathrm{C}$ im Vak., Aufnehmen des Rückstandes in $250 \mathrm{ml} P \ddot{A}$ und Filtration über $\mathrm{Na}_{2} \mathrm{SO}_{4}$. Das Filtrat wird anschließend mit 4,4 g Diäthylamin versetzt. Man läßt 30 Min. stehen, filtriert von den ausgefallenen Salzen über $\mathrm{Na}_{2} \mathrm{SO}_{4}$, zieht das Lösungsmittel ab und destilliert den Rückstand im Vak. zunächst über eine Vigreux-, dann über eine Drehbandkolonne. Ausb. 16,4 g IV $(53 \%)$.

\section{N,N-Diäthyl-3-(p-chlorphenyl-methyl-phenylsilyl)-propylamin (A) Rk. (5)}

Man bringt $15,5 \mathrm{~g}(0,05 \mathrm{Mol}) \mathrm{IV}$ und $18,3 \mathrm{~g}(0,25 \mathrm{Mol})$ Diäthylamin für $15 \mathrm{Stdn}$. im Bombenrohr bei $150^{\circ} \mathrm{C}$ zur Reaktion, versetzt das Reaktionsgemisch anschließend mit $150 \mathrm{ml} P \ddot{A}$, filtriert vom ausgefallenen $E t_{2} \mathrm{NH} \cdot \mathrm{HCl}$ über eine Schicht von $\mathrm{Na}_{2} \mathrm{SO}_{4}$, zieht das Lösungsmittel ab und fraktioniert im Vak. 2mal über eine Vigreux-Kolonne. In der Hauptfraktion finden sich $14,4 \mathrm{~g}$ A $(83 \%)$.

\section{$N, N$-Dimethyl-N'-(p-bromphenyl-methyl-phenylsilyl)-äthylendiamin (B)}

Rk. (8)

Man tropft zu $17,6 \mathrm{~g}(0,2 \mathrm{Mol}) \mathrm{N}, \mathrm{N}$-Dimethyläthylendiamin in $200 \mathrm{ml}$ $P \ddot{A}$ bei $20^{\circ} \mathrm{C}$ langsam und unter starkem Rühren eine Lösung von $15,6 \mathrm{~g}$ $(0,05 \mathrm{Mol})$ VI in $100 \mathrm{ml} P \ddot{A}$, wobei sofort das Hydrochlorid des Diamins ausfällt. Nach Beendigung des Zutropfens rührt man 1 Stde. bei $20^{\circ} \mathrm{C}$, dann 4 Stdn. unter Rückfluß weiter, filtriert vom Hydrochlorid, wäscht den Niederschlag mit jeweils $50 \mathrm{ml} P \ddot{A}$, zieht das Lösungsmittel ab, destilliert den Rückstand im Vak., nimmt die Hauptfraktion anschließend in $150 \mathrm{ml}$ $P \ddot{A}$ auf, filtriert über eine Schicht von $\mathrm{Na}_{2} \mathrm{SO}_{4}$ von erneut ausgefallenem Hydrochlorid, zieht das Lösungsmittel ab und fraktioniert im Vak. 2mal über eine Vigreux-Kolonne. Ausb. 12,9 g B (71\%).

\section{N,N-Diäthyl-N'-(p-bromphenyl-methyl-phenylsilyl)-äthylendiamin (G) Rk. (8)}

Analog zur Darstellung von $\mathrm{B}$ aus $23,2 \mathrm{~g} \mathrm{N,N-Diäthyl-äthylendiamin}$ und $15,6 \mathrm{~g}$ VI. Ausb. $14,3 \mathrm{~g}$ G $(73 \%)$. 
3. Chemische und physikalische Eigenschaften der neu dargestellten Verbindungen

Die Vorstufen I, V und VI waren bereits gut bekannt (Darstellung und Eigenschaften: $\left.I^{9}, V^{10},{ }^{12},{ }^{13}, V^{11}\right)$. Die von uns ermittelten Siedepunkte, Brechungsindices und Dichten stimmten weitgehend mit den Titeraturangaben überein ${ }^{2}, 3,5$.

Die neu dargestellten Vorstufen II-IV sind wasserklare, farblose Flüssigkeiten, die sich gut in den üblichen inerten organischen Solventien lösen. Sie sind mit Ausnahme von IV hydrolyseempfindlich.

II-IV wurden bisher noch nicht in der Literatur beschrieben; es wird lediglich über ein Gemisch stellungsisomerer Verbindungen des Typs $M e P h\left(\mathrm{C}_{6} \mathrm{H}_{4} \mathrm{Cl}\right) \mathrm{Si}\left(\mathrm{CH}_{2}\right)_{3} \mathrm{Cl}$ berichtet ${ }^{14}$, das durch Umsetzung eines entsprechenden Isomerengemisches von $\mathrm{MePh}\left(\mathrm{C}_{6} \mathrm{H}_{4} \mathrm{Cl}\right) \mathrm{SiH}$ mit Allylchlorid erhalten wurde.

$\mathbf{A}, \mathbf{B}$ und $\mathbf{C}$ sind ölige Flüssigkeiten, die sich gut in den gängigen organischen Lösungsmitteln wie $P \ddot{A}, E t_{2} \mathrm{O}, \mathrm{C}_{6} \mathrm{H}_{6}, \mathrm{CHCl}_{3}, \mathrm{CH}_{2} \mathrm{Cl}_{2}$ oder $\mathrm{CCl}_{4}$, dagegen schlecht in Wasser lösen. $\mathbf{A}$ ist bei einer Konzentration von $10^{-6} \mathrm{Mol} / 1$ in Tyrodelösung $\left(\mathrm{pH} 7,4,37^{\circ} \mathrm{C}\right)$ über mehr als 2 Stdn, stabil, während $\mathbf{B}$ und $\mathbf{C}$ unter den gleichen Bedingungen durch hydrolytische Spaltung der $\mathrm{Si}-\mathrm{N}$-Bindung offensichtlich so rasch zerfallen, daß im Gegensatz zu $A$ und den O-Isosteren keine pharmakologischen Effekte der $\mathrm{Si}-\mathrm{N}$-Verbindungen gemessen werden konnten (s. Kap. 5).

A, B und C sind im Vak. unzersetzt destillierbar. Bemerkenswert ist, daß bei der Destillation der 3-Chlorpropylsilane II-IV aus den Rohprodukten z.T. eine $\gamma$-Eliminierung auftritt. Diese ist vermutlich auf die Anwesenheit von Mg-Halogeniden und (oder) nicht umgesetztem Grignard-Reagens zurückzuführen. Die reinen Silane II-IV sind dagegen weitgehend unzersetzt im Vak. destillierbar.

\section{Zur Strukturermittlung der neu dargestellten Verbindungen}

wurden neben den Elementaranalysen (Tab. 1) vor allem ${ }^{1} \mathrm{H}-\mathrm{NMR}$ und Massenspektren herangezogen.

Die Kernresonanzspektren (Tab. 2) sind mit ihren vielen Signalgruppen auf Grund der charakteristischen chemischen Verschiebung und Multiplizität sehr nützlich und aussagekräftig. Die gemessenen Daten liegen weitgehend alle im Erwartungsbereich.

Die Massenspektren (Tab. 3) erwiesen sich auf Grund der charakteristischen Halogenisotopenmuster ebenfalls als sehr aussagekräftig. $\mathbf{B}$ und $\mathbf{C}$ zeigen in ihrem Fragmentierungsverhalten eine gewisse Verwandtschaft mit den $\mathrm{O}$-isosteren Verbindungen ${ }^{3-5}$. Bemerkenswert 
ist allerdings, daB in den Spektren von $\mathbf{B}$ und $\mathbf{C}$ neben den aus einer $\alpha$-Spaltung hervorgegangenen $\mathrm{CH}_{2}=\mathrm{NR}_{2}{ }^{\oplus}$-Ionen auch die entsprechenden $خ \mathrm{Si}-\mathrm{NH}=\mathrm{CH}_{2}{ }^{\oplus}$-Ionen auftreten. In den Spektren (RoutineMessungen) der 0 -Isosteren sind nur die $\mathrm{CH}_{2}=\mathrm{NR}_{2}{ }^{\oplus}$-Ionen auffindbar. Sie liefern in jedem Fall den Basispeak. Im Massenspektrum von A dominiert ebenfalls das durch $\alpha$-Spaltung entstehende $\mathrm{CH}_{2}=\mathrm{N}\left(\mathrm{C}_{2} \mathrm{H}_{5}\right)_{2}{ }^{\oplus}$-Ion.

Tabelle 1. Physikalische und elementaranalytische a Daten der neu dargestellten Verbindungen

\begin{tabular}{|c|c|c|c|c|c|c|}
\hline $\begin{array}{l}\text { Lfd. } \\
\text { Nr. }\end{array}$ & Summenformel b & $\begin{array}{c}\text { Sdp., } \\
{ }^{\circ} \mathrm{C} / \mathrm{T} \text { огт }\end{array}$ & $D_{4}^{20}$ & $n_{\mathrm{D}}^{20}$ & $\begin{array}{c}\% \mathrm{C} \\
\text { gef. } \\
\text { (ber.) }\end{array}$ & $\begin{array}{c}\% H \\
\text { gef. } \\
\text { (ber.) }\end{array}$ \\
\hline II & $\mathrm{C}_{9} \mathrm{H}_{10} \mathrm{Cl}_{4} \mathrm{Si}$ & $119-120 / 1$ & 1,3364 & 1,5489 & $\begin{array}{c}37,68 \\
(37,52)\end{array}$ & $\begin{array}{c}3,59 \\
(3,50)\end{array}$ \\
\hline III & $\mathrm{C}_{15} \mathrm{H}_{15} \mathrm{Cl}_{3} \mathrm{Si}$ & $155-157 / 0,01$ & 1,2448 & 1,5851 & $\begin{array}{c}53,84 \\
(54,64)\end{array}$ & $\begin{array}{c}4,46 \\
(4,59)\end{array}$ \\
\hline IV & $\mathrm{C}_{16} \mathrm{H}_{18} \mathrm{Cl}_{2} \mathrm{Si}$ & $155-157 / 0,2$ & 1,1457 & 1,5773 & $\begin{array}{c}61,65 \\
(62,13)\end{array}$ & $\begin{array}{r}5,75 \\
(5,87)\end{array}$ \\
\hline $\mathbf{A}$ & $\mathrm{C}_{20} \mathrm{H}_{28} \mathrm{CINSi}$ & $179-181 / 0,5$ & 1,0269 & 1,5490 & $\begin{array}{c}69,50 \\
(69,43)\end{array}$ & $\begin{array}{c}8,04 \\
(8,16)\end{array}$ \\
\hline B & $\mathrm{C}_{17} \mathrm{H}_{23} \mathrm{BrN}_{2} \mathrm{Si}$ & $154-155 / 0,1$ & 1,2000 & 1,5693 & $\begin{array}{c}55,07 \\
(56,19)\end{array}$ & $\begin{array}{c}6,27 \\
(6,38)\end{array}$ \\
\hline $\mathbf{C}$ & $\mathrm{C}_{19} \mathrm{H}_{27} \mathrm{BrN} \mathrm{N}_{2} \mathrm{Si}$ & $183-184 / 0,5$ & 1,1663 & 1,5585 & $\begin{array}{c}57,75 \\
(58,30)\end{array}$ & $\begin{array}{c}6,86 \\
(6,95)\end{array}$ \\
\hline
\end{tabular}

a Weitere elementaranalytische Daten sind in ${ }^{2}$ aufgeführt.

b Molgew. bestätigt durch Molpeak im Massenspektrum (Tab. 3).

Bemerkenswert ist, daß die Verbindungen II-IV im Massenspektrometer einer $\mathrm{C}_{3} \mathrm{H}_{6}$-Eliminierung (Cyclopropan oder Propylen?) unterliegen. Im Massenspektrum von $\mathrm{I}$ ergibt das $\mathrm{C}_{3} \mathrm{H}_{6}{ }^{\oplus} \cdot$. Ion sogar den intensivsten Peak und ist auch im Spektrum von II noch mit einer Intensität von $44 \%$ vorhanden. Dieses besondere Verhalten dürfte auf die gemeinsame 3-Chlorpropylgruppe zurückzuführen sein. In den Spektren von I-IV (Routine-Messungen) existieren keine Übergangssignale für eine elektronenstoßinduzierte $\mathrm{C}_{3} \mathrm{H}_{6}$-Eliminierung, so daß wir nicht mit Sicherheit sagen können, ob dieser Prozeß thermisch oder elektronenstoßinduziert ist.

\section{Pharmakologische Eigenschaften von A und B}

Die pharmakologischen Untersuchungen wurden in enger Zusammenarbeit am Institut für Pharmakologie und Toxikologie der Technischen Universität Braunschweig von H.-U. Rossée und F. Meyer durch- 
Tabelle 2. ${ }^{1} \mathrm{H}-\mathrm{NMR}-S p e k t r e n$ der neu dargestellten Verbindungen, Chemische Verschiebungen in $\delta[\mathrm{ppm}]$, Lösungsmittel: $\mathrm{CCl}_{4}$ (II-IV, A) bzw. $\mathrm{CDCl}_{3}$ (B und $\mathbf{C})$, Standard intern: TMS

\begin{tabular}{|c|c|c|c|c|}
\hline $\begin{array}{l}\text { Lfd. } \\
\text { Nr. }\end{array}$ & $\delta^{1} \mathrm{H}$ & Multiplizität & rel. Intensität & Zuordnung \\
\hline II & $\begin{array}{l}1,0-2,4 \\
3,48 \\
7,48^{a}\end{array}$ & $\begin{array}{l}\mathrm{m} \\
\mathrm{t} \\
\mathrm{m}\end{array}$ & $\begin{array}{l}4 \\
2 \\
4\end{array}$ & $\begin{array}{l}\mathrm{SiCH}_{2} \mathrm{CH}_{2} \mathrm{C} \\
\mathrm{CCH}_{2} \mathrm{Cl} \\
\mathrm{SiC}_{6} \mathrm{H}_{4} \mathrm{Cl}\end{array}$ \\
\hline III & $\begin{array}{l}1,0-2,3 \\
3,42 \\
7,0-7,8\end{array}$ & $\begin{array}{l}\mathrm{m} \\
\mathrm{t} \\
\mathrm{m}\end{array}$ & $\begin{array}{l}4 \\
2 \\
9\end{array}$ & $\begin{array}{l}\mathrm{SiCH}_{2} \mathrm{CH}_{2} \mathrm{C} \\
\mathrm{CCH}_{2} \mathrm{Cl} \\
\mathrm{SiC}_{6} \mathrm{H}_{5}, \mathrm{SiC}_{6} \mathrm{H}_{4} \mathrm{Cl}\end{array}$ \\
\hline IV & $\begin{array}{l}0,500 \\
0,7-2,1 \\
3,52 \\
7,0-7,8\end{array}$ & $\begin{array}{l}\mathrm{s} \\
\mathrm{m} \\
\mathrm{t} \\
\mathrm{m}\end{array}$ & $\begin{array}{l}3 \\
4 \\
2 \\
9\end{array}$ & $\begin{array}{l}\mathrm{SiCH}_{3} \\
\mathrm{SiCH}_{2} \mathrm{CH}_{2} \mathrm{C} \\
\mathrm{CCH}_{2} \mathrm{Cl} \\
\mathrm{SiC}_{6} \mathrm{H}_{5}, \mathrm{SiC}_{6} \mathrm{H}_{4} \mathrm{Cl}\end{array}$ \\
\hline $\mathbf{A}$ & $\begin{array}{l}0,50 \\
0,7-1,8 \\
0,94 \\
2,43 \\
2,37 \\
7,0-7,8\end{array}$ & $\begin{array}{l}\mathrm{s} \\
\mathrm{m} \\
\mathrm{t} \\
\mathrm{q} \\
\mathrm{t} \\
\mathrm{m}\end{array}$ & $\left\{\begin{array}{l}3 \\
10 \\
6 \\
9\end{array}\right.$ & $\begin{array}{l}\mathrm{SiCH}_{3} \\
\mathrm{SiCH}_{2} \mathrm{CH}_{2} \mathrm{C} \\
\mathrm{CCH}_{3} \\
\mathrm{NCH}_{2} \mathrm{C} \\
\mathrm{CCCH}_{2} \mathrm{~N} \\
\mathrm{SiC}_{6} \mathrm{H}_{5}, \mathrm{SiC}_{6} \mathrm{H}_{4} \mathrm{Cl}\end{array}$ \\
\hline $\mathbf{B}$ & $\begin{array}{l}0,54 \\
1,5^{\mathrm{b}} \\
1,9-3,1 \\
2,13 \\
7,1-7,8\end{array}$ & $\begin{array}{l}\mathrm{s} \\
\mathrm{m} \\
\mathrm{s} \\
\mathrm{m}\end{array}$ & $\begin{array}{r}3 \\
1 \\
10 \\
9\end{array}$ & $\begin{array}{l}\mathrm{SiCH}_{3} \\
\mathrm{NH} \\
\mathrm{NCH}_{2} \mathrm{CH}_{2} \mathrm{~N} \\
\mathrm{NCH}_{3} \\
\mathrm{SiC}_{6} \mathrm{H}_{5}, \mathrm{SiC}_{6} \mathrm{H}_{4} \mathrm{Br}\end{array}$ \\
\hline $\mathbf{C}$ & $\begin{array}{l}0,56 \\
0,96 \\
1,5^{\mathrm{b}} \\
2,1-3,2 \\
2,47 \\
7,1-7,8\end{array}$ & $\begin{array}{l}s \\
t \\
m \\
q \\
m\end{array}$ & $\begin{array}{l}3 \\
6 \\
1 \\
8 \\
9\end{array}$ & $\begin{array}{l}\mathrm{SiCH}_{3} \\
\mathrm{CCH}_{3} \\
\mathrm{NH} \\
\mathrm{NCH}_{2} \mathrm{CH}_{2} \mathrm{~N} \\
\mathrm{NCH}_{2} \mathrm{C} \\
\mathrm{SiC}_{6} \mathrm{H}_{5}, \mathrm{SiC}_{6} \mathrm{H}_{4} \mathrm{Br}\end{array}$ \\
\hline
\end{tabular}

a Zentrum eines symmetrischen Multipletts vom $A A^{\prime} B B^{\prime}$-System.

b Zentrum eines breiten Signals.

geführt. Für ausführliche Informationen sei auf die Dissertation Rossée verwiesen ${ }^{6}$.

A und B wurden am isolierten Meerschweinchen-Ileum in Hinblick auf ihre histaminolytischen, anticholinergen und muskulotrop spasmolytischen Eigenschaften untersucht. Während B überhaupt keine Wirkungen zeigt, sind bei $\mathbf{A}$ alle drei Wirkqualitäten vorhanden. Der Vergleich von A mit dem isosteren Sila-Clofenetamin zeigt jedoch, daß der Ersatz des O-Atoms durch eine $\mathrm{CH}_{2}$-Gruppe mit einem Verlust von etwa drei Vierteln der histaminolytischen Aktivität verbun- 
Tabelle 3. Einige charakteristische Schlüsselbruchstücke aus den Massenspektren der neu dargestellten Verbindungen. Probeneinführung durch

Direkteinlaß, Elektronenstoßionisierung mit $70 \mathrm{eV} .\left(\mathrm{R}=\mathrm{C}_{6} \mathrm{H}_{4} \mathrm{Cl}\right.$, $\left.\mathrm{R}^{\prime}=\mathrm{C}_{6} \mathrm{H}_{4} \mathrm{Br}, M e=\mathrm{CH}_{3}, E t=\mathrm{C}_{2} \mathrm{H}_{5}, P h=\mathrm{C}_{6} \mathrm{H}_{5}\right)$

\begin{tabular}{llccc}
\hline $\begin{array}{l}\text { Lfd. } \\
\text { Nr. }\end{array}$ & $m / e$ & $\begin{array}{c}\text { rel. } \\
\text { Intensität }\end{array}$ & $\begin{array}{c}\text { Isotopen- } \\
\text { muster }\end{array}$ & Ion \\
\hline
\end{tabular}

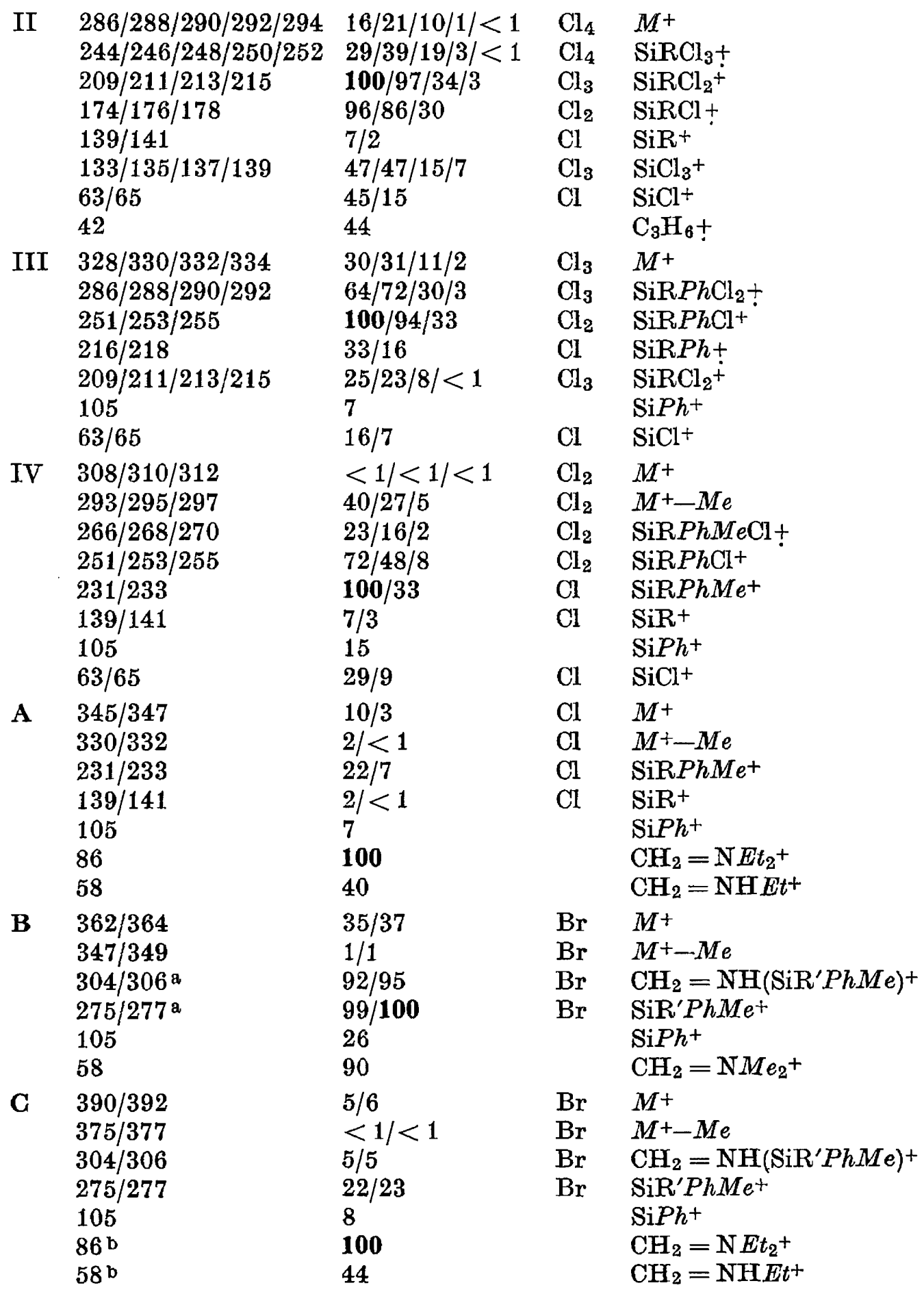

a Der Zerfall von $m / e=304 / 306 \mathrm{zu} m / e=275 / 277$ ist durch ein Übergangssignal bei $m / e=248,7 / 250,8$ belegt.

b Der Zerfall von $m / e=86 \mathrm{zu} m / e=58$ ist durch das entsprechende Übergangssignal bei $m / e=39,1$ belegt. 
den ist. Bezüglich der beiden anderen. Wirkqualitäten verhalten sich $\mathbf{A}$ und sein Isosteres fast gleich.

Während die früher von uns beschriebenen Sila-benzhydryläther und deren Derivate auf Grund ihres hydrolytischen Abbaus unter in-vitro-Bedingungen (Tyrode-Lösung, pH 7,4, $37^{\circ} \mathrm{C}$ ) nur eine kurze Wirkdauer besitzen, ist A über eine Meßdauer von 2 Stdn. stabil.

Da bei $\mathbf{B}$ eine pharmakologische Aktivität nicht meßbar ist, obwohl eine solche auf Grund der Isosterie von der Struktur her durchaus zu erwarten wäre, liegt die Vermutung nahe, daß die Verbindung durch hydrolytische Spaltung der Si-N-Bindung so rasch zerfällt, daß eine Verdrängung des Agonisten von den Rezeptoren nicht in ausreichendem Maße stattfindet.

Unser Dank gilt dem Verband der Chemischen Industrie, Frankfurt am Main, für die Unterstützung mit Sachmitteln und der Bayer-AG, Leverkusen, für die Überlassung von Chlorsilanen. Besonders danken wir Herrn Dr. H.-U. Rossée und Herrn Prof. Dr. F. Meyer für die pharmakologischen Untersuchungen an den neu dargestellten Verbindungen.

\section{Literatur}

1 5. Mitt.: R. Tacke und U. Wannagat, Mh. Chem. 107, 1265 (1976).

2 Mit Auszügen aus der Dissertation R. Tacke, Techn. Univ. Braunschweig 1974.

3 R. Tacke und $U$. Wannagat, Mh. Chem. 106, 1005 (1975).

4 R. Tacke und $U$. Wannagat, Mh. Chem. 107, 111 (1976).

5 R. Tacke und $U$. Wannagat, Mh. Chem. 107, 439 (1976).

6 Dissertation H.-U. Rossée, Techn. Univ. Braunschweig 1974.

$7 R$. Tacke und $U$. Wannagat, in Vorbereitung.

8 R. Tacke und $U$. Wannagat, Mh. Chem. 107, 449 (1976).

9 J.W.Ryan, G. K. Menzie und J.L. Speier, J. Amer. Chem. Soc. 82, $3601(1960)$.

10 J. F. Hyde und R. C. Delong, J. Amer. Chem. Soc. 63, 1194 (1941).

11 K. A. Andrianov, V.E. Nikitenkov und N.N. Sokolov, Izvest. Akad. Nauk SSSR, Otdel. Khim. Nauk 1960, 1224; Chem. Abstr. 55, 429 (1961).

12 A. D. Petrov, V. F. Mironov und V. G. Glukhovtsev, Izvest. Akad. Nauk SSSR, Otdel. Khim. Nauk 1954, 1123; Chem. Abstr. 49, 7510 (1955).

13 W. H. Daudt und J. F. Hyde, J. Amer. Chem. Soc. 74, 386 (1952).

14 Z. V. Belyakova, M. G. Pomerantseva und S. A. Golubtsov, Zh. Obshch. Khim. 35, 1048 (1965); Chem. Abstr. 63, 9978 (1965).

Korrespondenz und Sonderdrucke:

Dr. R. Tacke

Institut für Anorganische Chemie

Technische Universität Braunschweig

Pockelsstraße 4

D.3300 Braunschweig

Bundesrepublik Deutschland 